\title{
Cocaine Seeking During Initial Abstinence Is Driven by Noradrenergic and Serotonergic Signaling in Hippocampus in a Sex-Dependent Manner
}

\author{
Amy S Kohtz' and Gary Aston-Jones*,I \\ 'Brain Health Institute, Rutgers University and Rutgers Biomedical and Health Sciences, Piscataway, NJ, USA
}

\begin{abstract}
There is evidence for sex differences in cocaine addiction from both clinical and preclinical studies. In particular, preclinical studies indicate that females may be more sensitive than males to stress-induced drug seeking. The dorsal hippocampus $(\mathrm{DH})$ is prominently involved in the stress response, as are the locus coeruleus norepinephrine (LC-NE) and dorsal raphe serotonin (DR 5-HT) systems. Moreover, DH receives strong inputs from LC-NE and DR 5-HT neurons. We hypothesized that the stress associated with non-reinforced drug seeking during early abstinence (on extinction day I (EDI)) may contribute to drug seeking via $\beta$-adrenergic and $5-\mathrm{HT}$ neurotransmission in $\mathrm{DH}$. We observed decreased drug-seeking behavior on EDI following $10 \mathrm{mg} / \mathrm{kg}$ S-propranolol ( $\beta$-adrenergic and 5-HTIA/IB receptor antagonist), R-propranolol (5-HTIA/IB receptor antagonist), or racemic propranolol in both male and female rats. EDI increased Fos expression in DH, LC, and DR, and DH Fos was decreased by systemic S-propranolol. Based on these results, we investigated the effects of blocking 5-HT and $\beta$-adrenoceptor transmission in DH on drug seeking during EDI by infusing a cocktail of WAY I 00635 plus GR I 27935 (5-HTIA/IB receptor antagonists), betaxolol plus ICl-II 855 I ( $\beta$ | and $\beta 2$ antagonists), or S-propranolol alone. In males, WAYI00635/ GRI27935 was most effective in reducing drug-seeking on ED I, whereas betaxolol//Cl-1 I 855 I was ineffective. In contrast, S-propranolol was most effective in females in reducing drug seeking on EDI, and WAYI00635/GRI27935 and betaxolol/ICl-1 I855 I were each partially effective. Our results indicate that drug seeking during initial abstinence involves 5 -HT and $\beta$-adrenergic signaling in female DH, but only 5-HT signaling in male DH.

Neuropsychopharmacology (2017) 42, 408-4I8; doi:I0.1038/npp.2016.150; published online I4 September 2016
\end{abstract}

\section{INTRODUCTION}

Both clinical and rodent studies reveal notable sex differences in cocaine abuse. Women acquire cocaine dependence earlier and more rapidly, show increased craving and severity of cocaine use, and relapse to cocaine more rapidly as compared with men (Kosten et al, 1993; Robbins et al, 1999; Becker and $\mathrm{Hu}, 2008)$. In addition, women report more depression and anxiety disorders after prolonged cocaine use (Griffin et al, 1989). Several measures of cocaine dependence are greater in female rodents, including self-administration, drug seeking, higher progressive ratio breakpoints, and reinstatement of extinguished cocaine seeking (Lynch and Taylor, 2004; Kippin et al, 2005; Feltenstein et al, 2011; Zhou et al, 2012). The biological mechanisms for these sex differences remain unclear.

Growing evidence indicates that sex differences in drugseeking behavior may be mediated by differences in stress reactivity. Stress is an established risk factor for relapse

* Correspondence: Dr G Aston-Jones, Brain Health Institute, Rutgers University, RWJMS Research Building Room 259, 683 Hoes Lane West, Piscataway, NJ 08854, USA, Tel: + I 732235 4659, Fax: + I 732235 58|4, E-mail: gsa35@ca.rutgers.edu

Received 20 January 2016; revised 2 August 2016; accepted 7 August 2016; accepted article preview online 12 August 2016
(Sinha, 2001, 2008), and female rats display higher serum corticosterone levels following various stressors (Tinnikov, 1999; Lu et al, 2015). Notably, locus coeruleus norepinephrine (LC-NE) neurons in females are more sensitive to the stress-related neuropeptide corticotropin-releasing factor (CRF; Curtis et al, 2006), and conversely dorsal raphe serotonin (DR-5-HT) neurons are more sensitive to CRF in males (Howerton et al, 2014). Moreover, CRF- or yohimbine-induced reinstatement of cocaine seeking is more robust in female compared with male rats (Feltenstein $e t$ al, 2011; Buffalari et al, 2012). These data together indicate that sex differences in stress responding may influence drug seeking.

The dorsal hippocampus (DH) is a focal region in stress circuitry, and receives strong inputs from LC-NE, DR-5-HT, and ventrolateral periaqueductal gray (VLPAG) 5-HT neurons. The $\mathrm{DH}$ has a number of structural and biochemical sex differences, including CRF receptor binding affinity, serotonin synthesis, and adrenergic-, corticosterone-, and GABA-receptor expression (Turner, 1992; Madeira and Paula-Barbosa, 1993; Galea et al, 1997; Rhodes and Rubin, 1999; Shors et al, 2001). Thus, the DH may be involved in sexrelated stress-modulated behaviors such as drug seeking.

Propranolol was effective in animal studies to attenuate withdrawal anxiety and associated drug seeking (Harris and 
Aston-Jones, 1993; Smith and Aston-Jones, 2008), and propranolol and carvedilol have had promising effects in clinical studies to reduce cocaine-seeking behaviors (Sofuoglu et al, 2000; Kampman, 2009). Although these drugs are generally used as $\beta$-adrenergic antagonists, propranolol is also a 5-HT1A/1B antagonist (Pazos et al, 1985). Our lab previously found that 5-HT signaling is involved in drug reward (Harris et al, 2001; Harris and Aston-Jones, 2001), and we hypothesize that some of the effects of propranolol to influence drug seeking may also involve antagonism of 5-HT receptors. In the following studies, we utilized S-propranolol, an adrenergic and serotonin antagonist, and its enantiomer R-propranolol that only antagonizes 5-HT receptors, to probe the relative involvement of signaling at these receptors in drug-seeking behaviors.

The initiation of abstinence when expected drug is not available (extinction day 1 (ED1)) represents a stressful time point marked by increased drug craving that may be due to changes in drug contingency. Cravings on ED1 may result in negative reinforcement by subsequent administration of drug, and conditioning to avoid the aversive consequences of drug absence (Le Moal and Koob, 2007). We hypothesized that these factors may make the conditions surrounding the initiation of abstinence important for drug seeking and subsequent relapse. In humans, increases in drug craving during initial abstinence can predict the likelihood of relapse over a 30-day period (Weiss et al, 2010), and drug craving can be triggered by exposure to contextual stimuli associated with selfadministered drugs (Wikler, 1973; O'Brien et al, 1992). Here, we tested the roles of $\beta$-adrenoceptors and 5-HT1 receptors in $\mathrm{DH}$ in sex differences in early abstinence (ED1) responding.

\section{MATERIALS AND METHODS}

\section{Subjects}

Male (300-450 g, $n=108)$ and female (225-300 g, $n=65)$ Sprague Dawley rats (Charles River Laboratories) were singly housed under a reversed $12 \mathrm{~h} / 12 \mathrm{~h}$ light/dark cycle (lights off at $0600 \mathrm{~h}$ ); all experiments were during the active cycle. Rats had free access to food and water and were housed in the animal facility at the Medical University of South Carolina (AAALAC accredited). All experiments were approved by the institutional animal care and use committee and conducted in accordance to the National Institutes of Health specifications outlined in their Guide for the Care and Use of Laboratory Animals.

\section{Jugular Catheter Surgeries}

Animals were anesthetized with ketamine/xylazine $(56.5 / 8.7 \mathrm{mg} / \mathrm{kg})$ and given meloxicam $(1 \mathrm{mg} / \mathrm{kg})$ as an analgesic. Chronic in-dwelling catheters were constructed in-house and inserted as described previously (Smith et al, 2009). Animals were given cefazolin (10 mg, intravenous (i.v.)) and heparin (10 U, i.v.) starting 3 days following surgery and daily following self-administration sessions. Rats recovered from surgery for at least 1 week before selfadministration training.

\section{Dorsal Hippocampus Cannulae}

Immediately following jugular catheter surgery, rats were placed in a stereotaxic apparatus and bilateral guide cannulae were implanted targeted to $\mathrm{DH}(\mathrm{AP}:-3.0, \mathrm{ML}: \pm 2.0, \mathrm{DV}$ : -2.5). Cannula placements were confirmed for each rat following completion of behavioral analysis (Figure 2e).

\section{Drugs}

Cocaine hydrochloride (NIDA, Research Triangle Park, NC) was dissolved in $0.9 \%$ sterile saline. S-propranolol (S-prop; combined $\beta$-adrenoceptor and 5-HT1A/1B receptor antagonist; Pazos et al, 1985), R-propranolol (R-prop; enantiomer that blocks 5 -HT1A/1B but not $\beta$-adrenoceptors), and the $\mathrm{R} / \mathrm{S}$ racemic propranolol mixture (R/S-prop) were all administered intraperitoneally (i.p.) at a dose of $10 \mathrm{mg} / \mathrm{kg}$ 30 min before testing in ED1. A cocktail of WAY100635 plus GR127935 (5-HT1A/1B receptor antagonists respectively, $1 \mathrm{nmol} / 1.0 \mu \mathrm{l} /$ Side, WAY/GR), betaxolol plus ICI-118 551 ( $\beta 1$ and $\beta 2 \mathrm{AR}$ antagonists, 1 or $3 \mathrm{nmol} / 1.0 \mu \mathrm{l} / \mathrm{side}$, Bet/ICI), or S-prop ( $8.43 \mathrm{nmol} / 1.0 \mu \mathrm{l} / \mathrm{side})$ were intracranially administered at a rate of $0.25 \mu \mathrm{l} / \mathrm{min}$ over 4 and $10 \mathrm{~min}$ before testing in ED1. All drugs were dissolved in $0.9 \%$ saline and were obtained from Tocris Sciences.

\section{Cocaine Self-Administration}

Self-administration sessions were conducted in standard operant chambers housed in sound-attenuating cubicles and controlled via MED-PC IV software (Med-Associates, St Albans, VT) as described previously (Smith and Aston-Jones, 2011). All rats were trained to press an active lever for cocaine reward (i.v. infusion of cocaine hydrochloride) for at least 10 days on a fixed ratio 1 (FR1) schedule of reinforcement in $2 \mathrm{~h}$ sessions to reach a criterion of $>10$ cocaine infusions/day $(0.2 \mathrm{mg} / 50 \mu \mathrm{l}$ infusion for males, $0.16 \mathrm{mg} / 50 \mu \mathrm{l}$ infusion for females). Each active press results in one infusion, followed by a $20 \mathrm{~s}$ time-out period in which lever pressing had no reward or cues. An inactive lever was also present; presses on it were tabulated but had no consequence.

\section{Cocaine Seeking During ED1}

At $24 \mathrm{~h}$ after the final self-administration session rats were exposed to the self-administration chamber for $90 \mathrm{~min}$ during which time presses on either lever had no consequence. Cue-induced drug seeking was not examined as our studies focused specifically on the role of $\mathrm{DH}$ in drug seeking during ED1. Active lever pressing during ED1 was indicative of drug-seeking behavior, as previously reported (Feltenstein and See, 2007).

\section{Locomotor Behavior}

At 1 week following ED1 testing, rats were acclimated to locomotor chambers for $2 \mathrm{~h}$ /day for 2 days before testing. Locomotor chambers were clear acrylic chambers $(\sim 40 \times 40 \times 30 \mathrm{~cm})$ equipped with Digiscan monitors (AccuScan Instruments) containing a $16 \times 16$ photobeam array. Photobeam breaks were recorded by DigIPRO software (Version 1.4). Rats were microinfused with antagonists into $\mathrm{DH} 10 \mathrm{~min}$ before a $120 \mathrm{~min}$ test session. Rats were tested for saline, WAY/GR, Bet/ICI, or S-prop in a counterbalanced manner, with tests at least 2 days apart. 


\section{Tissue Collection}

At 15 min following the ED1 session, rats were deeply anesthetized and transcardially perfused using $0.9 \%$ saline followed by $4 \%$ paraformaldehyde. Brains were collected, post-fixed for $24 \mathrm{~h}$ in $4 \%$ paraformaldehyde, transferred to a $20 \%$ sucrose solution, and stored at $4{ }^{\circ} \mathrm{C}$. Coronal sections ( $40 \mu \mathrm{m}$ thick) were cut using a cryostat and processed for single- or double-label immunohistochemistry. A separate group of rats were given i.p. injections of saline $24 \mathrm{~h}$ after the final self-administration session and returned immediately to their home cage. After $2 \mathrm{~h}$, rats were killed and brains processed for immunohistochemistry.

\section{Fos Immunohistochemistry}

Immunohistochemistry was carried out as previously described (see, eg, Mahler and Aston-Jones, 2012). The Fos antibody was used at 1:10 000 (Calbiochem, Santa Cruz, CA, Catalog PC38, Lot D00148958). The 5-HT antibody was used at 1:5000 (IncStar, Stillwater, MN, Catalog 20079). Detailed descriptions of procedures are included in the Supplementary Methods.

\section{Corticosterone Assay}

Eight rats/sex were used, and serum was obtained via tail vein blood draw. Within-subjects sampling was taken at a no-cocaine baseline, homecage condition ( $24 \mathrm{~h}$ abstinence), and at maximal responding during the ED1 session (30 $\mathrm{min})$. Serum was centrifuged (10 $000 \mathrm{RPM}$ at $\left.4{ }^{\circ} \mathrm{C}\right)$ and plasma was reserved for corticosterone processing. Plasma corticosterone levels were measured using an enzyme immunoassay kit (Enzo Life Sciences).

\section{Statistical Analysis}

Independent $t$-tests, Pearson's $R$ correlations, or one- or twoway analysis of variance (ANOVA) were used to compare differences between groups in responding or neuronal activation. Individual statistics for all results are listed in Supplementary Table S1 (behavior) and Supplementary Table S2 (immunohistochemistry).

\section{RESULTS}

\section{Sex Differences in FR1 Self-Administration and Cocaine Seeking on ED1}

Analyses of FR1 responding over the first 10 consecutive training days revealed significant sex differences in active lever pressing, and number of infusions of cocaine, but not in inactive lever presses. These data indicate that there were sex differences in number of cocaine-cue pairings, but these differences occurred during only the first 3 days of selfadministration. Thus, females may be highly motivated to respond for cocaine during early training compared with males (Figure 1a). Total $\mathrm{mg} / \mathrm{kg} /$ day cocaine intake was higher in females compared with males on all days (Figure 1b).

Active lever pressing during the ED1 session was greater than during self-administration in both sexes. Moreover, females had higher active lever pressing on ED1, and a greater increase in lever pressing on ED1 compared with selfadministration than males (Figure 1c). However, this did not correlate to total intake during self-administration or to number of cocaine-cue pairings in males or females (Table 1). We then analyzed active lever pressing in $5 \mathrm{~min}$ epochs during the ED1 session, and found that the nearly all active lever pressing occurred during the first $25 \mathrm{~min}$, and decreased gradually over time in both males and females (Figure 1d). Females showed greater early responding as well as greater extinction resistance within session (Figure 1d).

Plasma from within-subjects sampling revealed that ED1 substantially increased circulating corticosterone compared with either no-cocaine or homecage groups, similarly in males and females (Figure 1e). In addition, circulating corticosterone correlated with ED1 lever responding in both sexes (Table 1; Supplementary Figure S2E).

\section{Propranolol Decreased Cocaine Seeking During ED1 in Male and Female Rats}

Pretreatment with $10 \mathrm{mg} / \mathrm{kg}$ i.p. S-prop, R-prop, or S/R-prop reduced drug seeking on ED1 compared with saline controls (Figure 2a). The combined $\beta$-AR and 5-HT antagonists $\mathrm{S}$-prop and $\mathrm{S} / \mathrm{R}$-prop were more effective in reducing active lever pressing on ED1 in females than in males (Supplementary Table S1). Pretreatment with these combined $\beta$-AR +5 -HT antagonists, or with the 5-HT antagonist R-prop, was similarly effective in males (Supplementary Table S1), indicating that 5 -HT but not $\beta$-adrenergic signaling is important for drug seeking in males. In females, combined 5 -HT $+\beta$-AR antagonists (S-prop and S/R-prop) tended to be more effective than serotonin antagonists alone (R-prop; Figure 2a). Thus, systemic treatments indicate that drug seeking in male rats involves 5 -HT, but little or no $\beta$-AR signaling, whereas drug seeking in females involves signaling at both 5 -HT and $\beta$-AR receptors.

\section{Fos Induction in DH Following ED1 Exposure Was Blocked by Systemic S-Prop}

Rats tested in the prior experiment were killed $15 \mathrm{~min}$ after the ED1 session and brains were examined for Fos expression in $\mathrm{DH}$ as an index of neuronal stimulation during ED1. Fos expression in $\mathrm{DH}$ increased during ED1 testing compared with homecage controls (Figure 3). Administration of S-prop decreased Fos expression in $\mathrm{DH}$ to homecage levels. In the CA1 region (Figure $3 \mathrm{a}$ and $\mathrm{d}$ ), females had higher Fos expression following ED1 compared with males. CA1 Fos expression significantly correlated to ED1 active lever pressing among females, but not males, and when collapsed across sex (Table 1 and Supplementary Figure S2A). In CA3 and dentate gyrus (DG; Figure 3b, c, e and f), ED1 exposure increased Fos irrespective of sex, and Fos was decreased by pretreatment with systemic S-prop. Fos expression in CA3 correlated with active lever pressing on ED1 when collapsed across sex, but not when analyzed for either sex separately (Table 1). Fos expression in DG did not correlate to active lever pressing on ED1 when collapsed across sex or when analyzed separately (Table 1). Thus, Fos induction implicates the DH in drug seeking during ED1. 
Intra-Hippocampus Administration of S-Prop, WAY/GR, or Bet/ICI Reduced ED1 Cocaine Seeking in a Sex-Dependent Manner

We used local microinfusions of 5-HT or $\beta$-AR antagonists into $\mathrm{DH}$ to investigate possible sexually dimorphic roles of these receptors in drug seeking on ED1. Intra-hippocampal antagonism of 5-HT1A/1B receptors by a WAY/GR cocktail, or antagonism of $5-\mathrm{HT} 1 \mathrm{~A} / 1 \mathrm{~B}$ plus $\beta$-AR receptors by S-prop, significantly reduced drug-seeking behavior on ED1 (active lever presses) as compared with inhibition of $\beta$-ARs alone by the Bet/ICI cocktail in males and females (Figure 2b). In females, S-prop or WAY/GR into DH was more effective in reducing ED1 active lever presses compared with Bet/ICI. Bet/ ICI into DH was ineffective in reducing active lever presses in male rats, and was comparable to saline-administered controls. Both WAY/GR and S-prop into DH were effective in reducing lever presses in males (statistics in Supplementary Table S1).

A follow-up study in males tested whether the ineffectiveness of Bet/ICI was due to sex differences in antagonist sensitivity. We found that Bet/ICI was also ineffective at a threefold higher dose $(3 \mathrm{nmol} / 1.0 \mu \mathrm{l} /$ side $)$ at reducing ED1 responding in males (Supplementary Figure S1A), but significantly reduced memory performance in an object recognition task (Supplementary Figure S1B). Thus, intrahippocampal infusions indicate that 5-HTRs in $\mathrm{DH}$ are involved in ED1 drug seeking in male and female rats, and $\beta$-ARs are also involved in females but not in males.

\section{Motor Effects of Intra-Hippocampal S-Prop, WAY/GR, or Bet/ICI}

We examined the effects of the intra-hippocampal compounds on motor activity in an open field (Figure $2 c$ and d). There was no significant effect of drug condition to influence locomotor behavior in females (Figure 2c). There was a significant main effect of drug condition on locomotor behavior in males, wherein Bet/ICI reduced motor activity compared with saline controls (Figure 2d). However, as there were no effects of Bet/ICI to reduce $\mathrm{ED} 1$ pressing in males, we conclude that motor effects of these agents do not contribute to their effects on ED1 behavior.

\section{ED1 Exposure Increased the Percentage of Fos-Expressing Neurons in Locus Coeruleus and Serotonin Nuclei}

Dorsal raphe nucleus (DRN) is a source of 5-HT innervation of DH (Wirtshafter et al, 1986); therefore, we examined Fos a
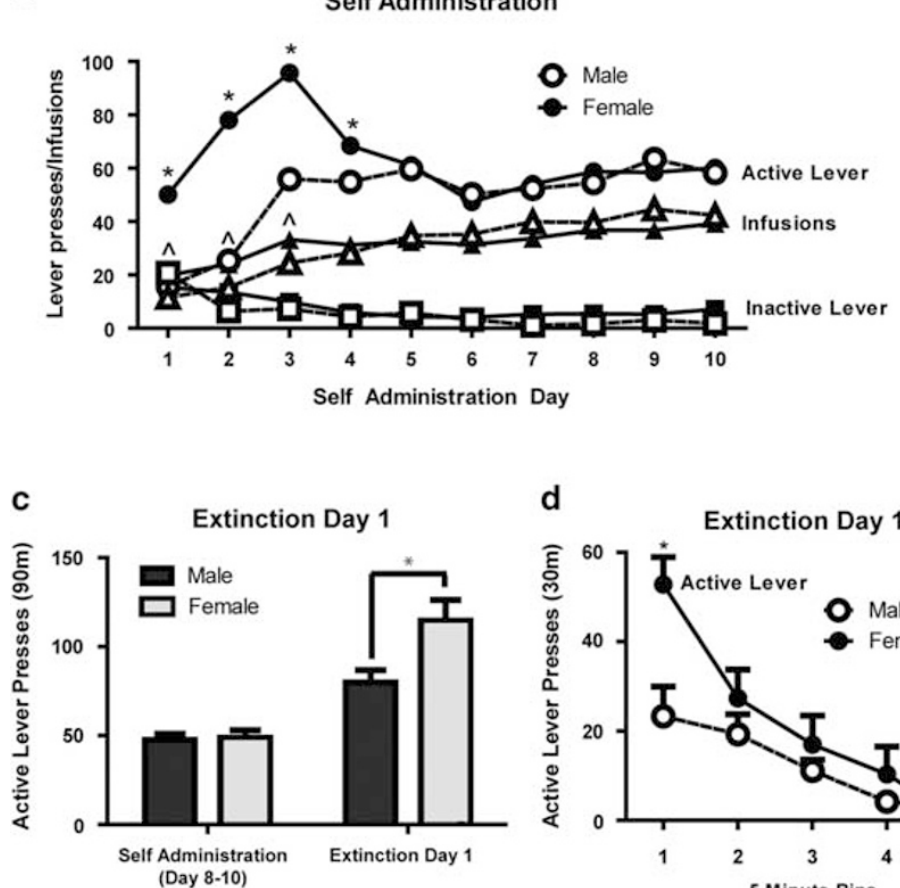

d

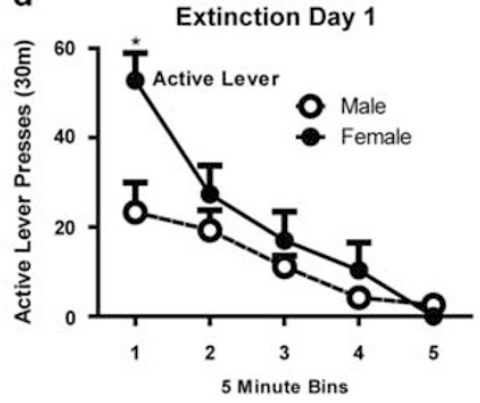

b

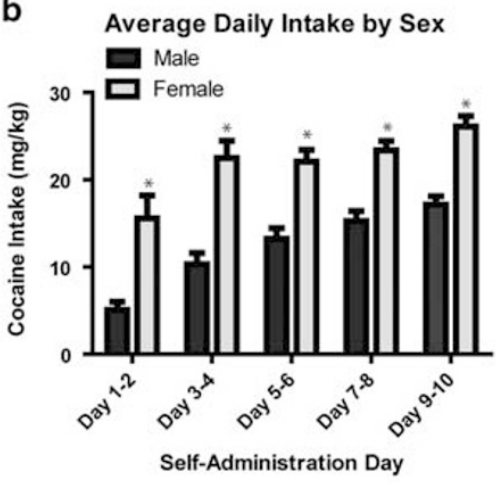

e Corticosterone

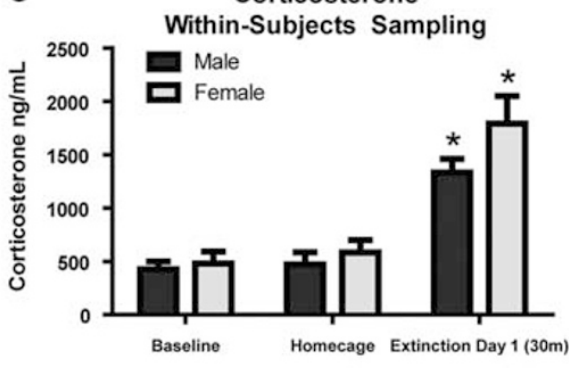

Figure I Sex differences in FRI acquisition ( $n=50-52 /$ group) and drug seeking on EDI ( $n=13-24 /$ group). Two-way ANOVAs were performed between sex and condition followed by post hoc $t$-tests for all analyses. Comprehensive statistics are provided in Supplementary Table SI. (a) Self-administration data for FRI days I-I0. Active lever presses are indicated as circles, infusions as triangles, and inactive lever presses as squares. Open shapes indicate females and filled shapes indicate males. There was a significant main effect of sex to influence active lever pressing for cocaine reward, and a significant interaction between sex and time. The post hoc tests showed individual days when females pressed significantly more than males to obtain cocaine reward (days $1-4$; * $p<0.05$ ) and when females acquired more cocaine infusions (days I-3; ^ $p<0.05$ ). (b) Average daily intake for male and female rats corrected for daily body weight and expressed as average $\mathrm{mg} / \mathrm{kg}$ in 2-day epochs. Females infused more $\mathrm{mg} / \mathrm{kg}$ of cocaine daily compared with males; $* p<0.05$. (c) FRI and extinction day I (EDI) active lever presses in male and female rats. There was a significant main effect of EDI, and interaction between EDI and sex, for active lever pressing. Both male and female rats had greater lever presses on EDI compared with FRI self-administration, and females had greater EDI lever pressing than males; * $p<0.05$. (d) EDI data in male and female rats in 5 min bins for minutes $0-25$ ( $n=6-14 /$ group). There was a significant interaction between time and sex wherein females displayed higher drug-seeking behavior during the initial $5 \mathrm{~min}$ bin; $* p<0.05$. (e) Corticosterone analyses sampled within subjects at a nococaine baseline (baseline), homecage condition ( $24 \mathrm{~h}$ withdrawal), or after $30 \mathrm{~min}$ of EDI responding $(n=8 /$ sex). EDI exposure increased corticosterone levels in males and females compared with no-cocaine or homecage conditions; $* p<0.05$. 


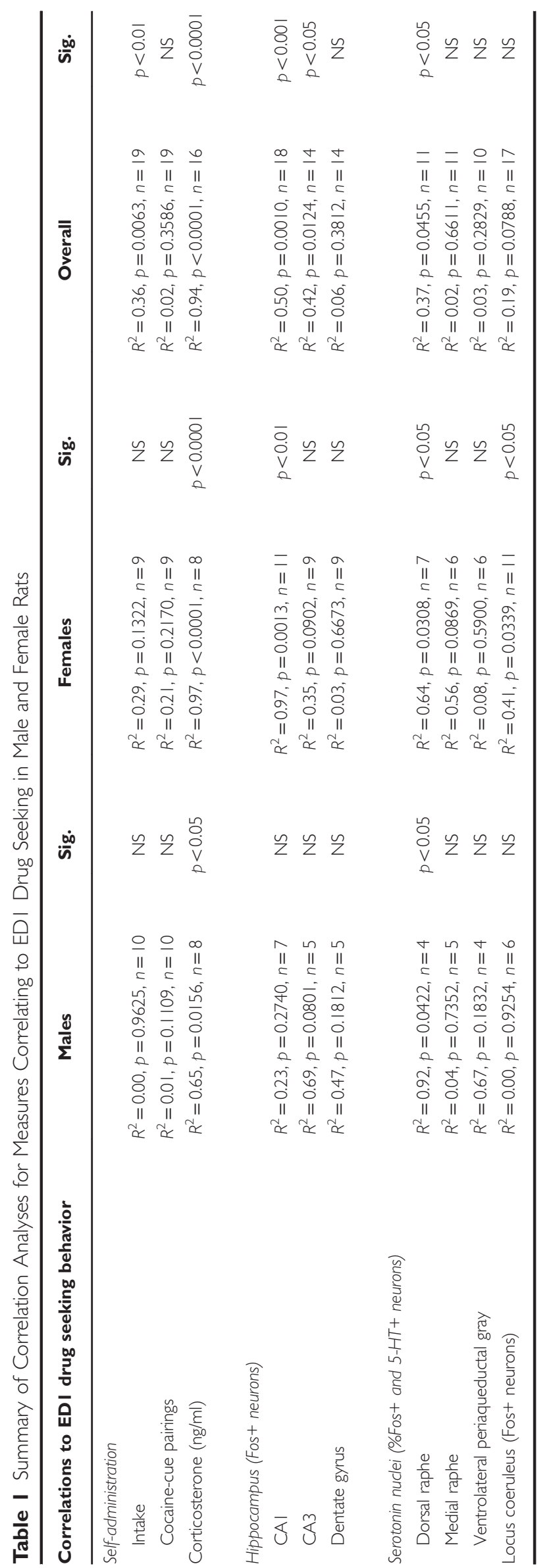

expression in DRN. The percentage of DRN 5-HT neurons that were Fos+ positively correlated with ED1 active lever pressing in both males and females, and when analyzed together (Table 1 and Supplementary Figure S1A). These results are consistent with our behavioral effects, indicating that 5-HT in the DRN plays a role in drug-seeking behavior on ED1 in both male and female rats.

We similarly analyzed Fos expression in 5-HT neurons in VLPAG nearby DRN. The percentage of 5-HT neurons in VLPAG that were Fos+ on ED1 was greater than homecage controls in both sexes. Moreover, ED1 females had a higher percentage of 5-HT neurons in VLPAG that were Fos+ and a greater increase compared with their respective homecage controls than males (Figure 4b). Thus, 5-HT activity in the VLPAG may also play a role in drug-seeking behavior on ED1, but more prominently in females.

Results also indicated that there are sex differences in the percentage of MRN 5-HT neurons that are Fos+, wherein more MRN 5-HT neurons are Fos+ in males (but not females) in response to ED1 compared with HC (Figure 4c). No correlations between 5-HT Fos in MRN and active lever pressing were observed (Table 1). Thus, 5 -HT activity in the MRN may also play a role in drug-seeking behavior on ED1, but more prominently in males.

We also found that there are sex differences in LC Fos, wherein increased Fos induced by ED1 compared with HC controls is greater in females compared with males (Figure 4d). LC Fos also positively correlated to ED1 active lever pressing in females, but not males, and not when analyzed collapsed across sex (Table 1 and Supplementary Figure S1D). These results are consistent with our behavioral effects, indicating that the LC plays a role in drug-seeking behavior on ED1 particularly in female rats.

\section{DISCUSSION}

Our results indicate that hippocampal 5-HT1A/1B and $\beta$-adrenergic receptors are involved in ED1 cocaine-seeking behavior. Systemic administration of S-prop or S/R-prop ( $\beta$-AR and $5-\mathrm{HT} 1 \mathrm{~A} / 1 \mathrm{~B}$ receptor antagonists), or of the enantiomer R-prop (a 5-HT1A/1B receptor antagonist without $\beta$-AR antagonism), decreased ED1 drug-seeking behavior in both males and females. This may indicate that systemic propranolol modulates drug seeking on ED1 at least in part via 5-HT receptors. Local microinjection studies with specific antagonists in hippocampus confirm this possibility, and indicate prominent sex differences in the involvement of specific receptor subtypes on ED1. Notably, antagonism of hippocampus 5-HT receptors reduced ED1 drug seeking in both males and females, but hippocampus $\beta$-AR receptor antagonism was only effective in females. These data indicate that ED1 responding is strongly modulated in male DH by 5 -HT receptors, whereas in females both 5 -HT and $\beta$-ARs are involved. These findings also indicate that $\mathrm{DH}$ may be a focal point in brain for sex differences in drug seeking during early abstinence.

\section{Sexually Dimorphic Noradrenergic and Serotonergic Neuron Activation on ED1}

Our behavioral results corresponded to neural activation of 5-HT and NE neurons in nuclei that project to $\mathrm{DH}$. We 
a

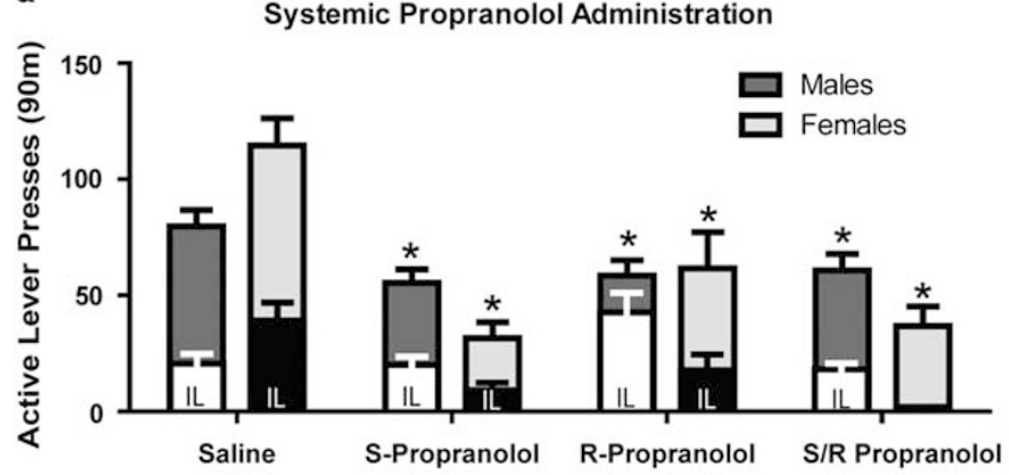

b Intrahippocampal manipulations on Extinction Day 1
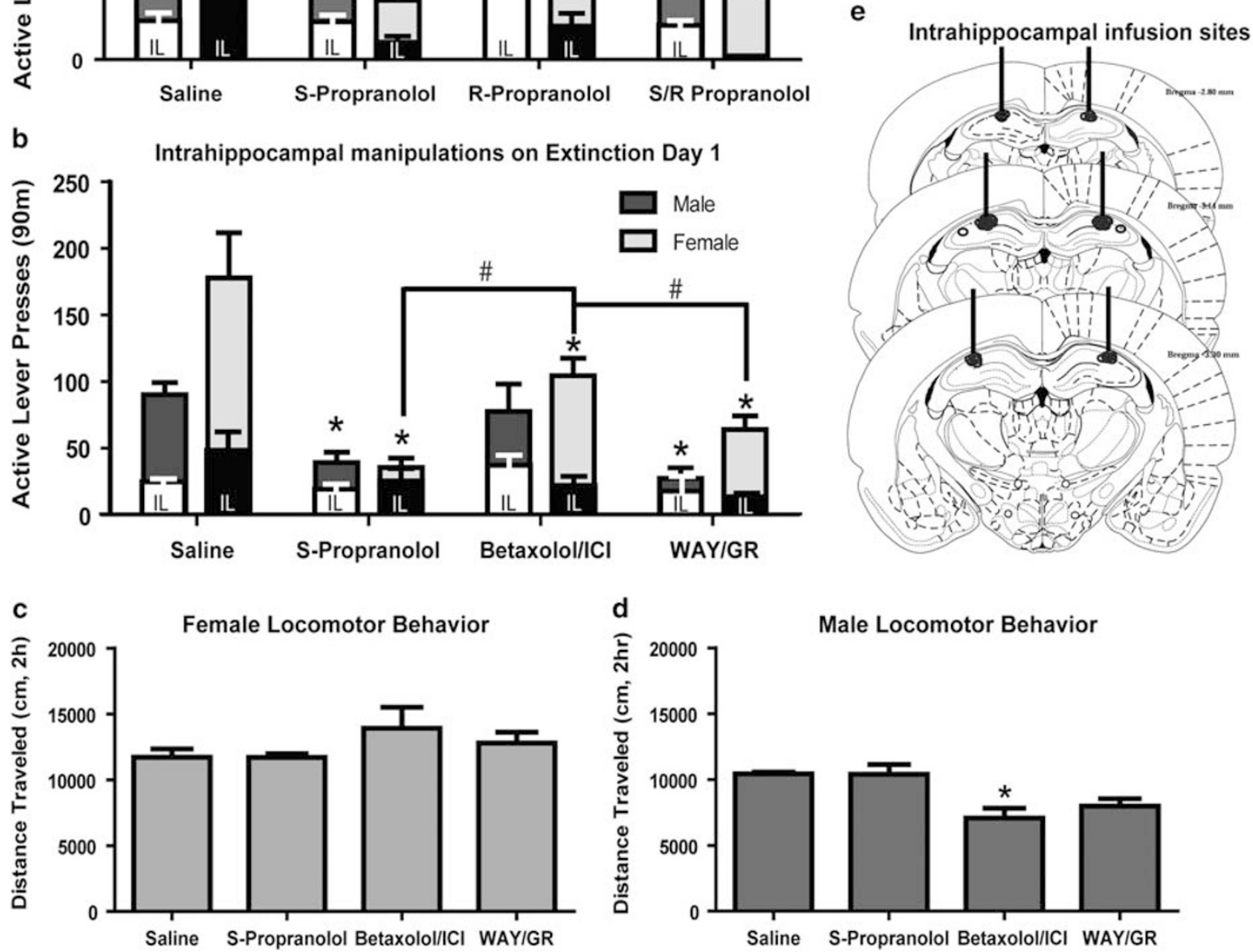

Figure 2 EDI drug seeking is driven by 5-HT receptors in males and 5-HT and $\beta$-adrenergic receptors in females. Inactive lever presses are in white (males) and black (females). Two-way ANOVAs were performed between sex and condition followed by post hoc t-tests for all analyses unless otherwise indicated. Statistics for all panels are provided in Supplementary Table SI. (a) There was a main effect of drug condition on EDI drug-seeking behaviors in male and female rats ( $n=8-24 / g r o u p)$. The post hoc tests revealed that all three drugs were effective in reducing EDI drug seeking compared with saline vehicle controls; ${ }^{*} p<0.05$. There was a significant interaction between sex and drug condition wherein S-propranolol and S/R propranolol were more effective in females compared with males. IL indicates inactive lever bars. (b) Intra-hippocampus infusions of $\beta$-adrenergic and 5-HTIA/IB receptor antagonists influence drug seeking in a sex-dependent manner ( $n=6-10 /$ group). There was a significant main effect of sex, and significant main effect of drug condition on EDI drug seeking. The post hoc tests reveal S-propranolol and WAY/GR were effective in reducing drug seeking in males and females, and betaxolol/ICl was also effective in females only; ${ }^{*} p<0.05$. There was also a significant interaction between sex and drug condition. ${ }^{\#}$ Significant post hoc differences between drug conditions in females only $p<0.05$, wherein S-propranolol and WAY/GR compounds were more effective than betaxolol/ICl cocktail in females. IL indicates inactive lever. (c) There were no significant effects of intra-hippocampal infusions on locomotor activity in females (one-way ANOVA for drug treatment; $n=6 /$ group). (d) There was a significant main effect of drug condition on locomotor activity for intra-hippocampal infusions in male rats (one-way ANOVA; $n=6 /$ group); $* p<0.05$. (e) Histological confirmation of intra-hippocampal infusion sites. Injection sites are indicated by open circles, and cannula tracts are marked as bars (Paxinos and Watson, 2006).

observed an increased percentage of DR 5-HT neurons that were Fos+ on ED1 as compared with homecage controls that correlated to drug-seeking behavior in both sexes. In addition, LC Fos activation increased on ED1 and correlated to ED1 drug seeking in females only. Although we only measured total Fos+ neurons in LC, nearly all LC neurons in the region examined are
Fos+ (Dahlstrom and Fuxe, 1964).There are also no differences in overall LC size between males and females (Babstock et al, 1997). As such, we concluded that Fos alone was sufficient to represent LC-NE neuron signaling and was comparable across sexes. One caveat is that our homecage group ( $24 \mathrm{~h}$ withdrawal) may have reduced Fos+ neurons in DH and DR compared with 
a

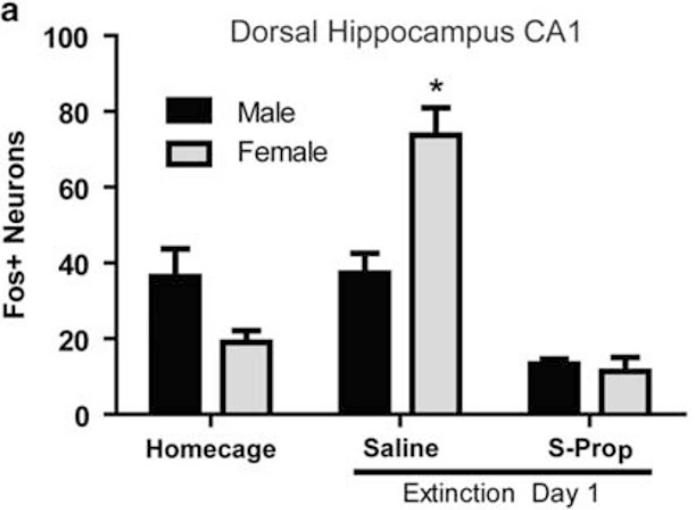

b

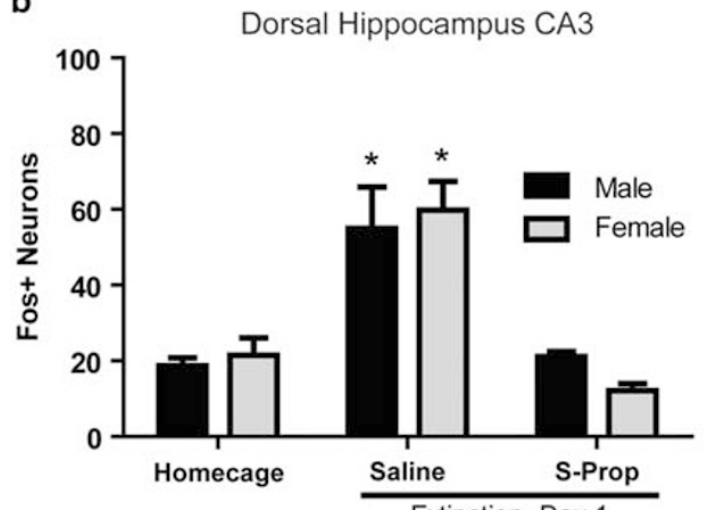

Extinction Day 1

c

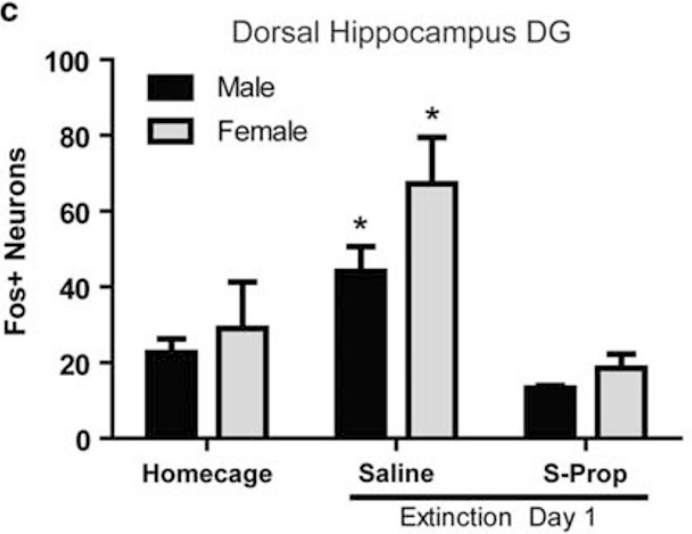

d Depiction of the $\mathrm{DH}$ areas where Fos immunoreactive cells were counted

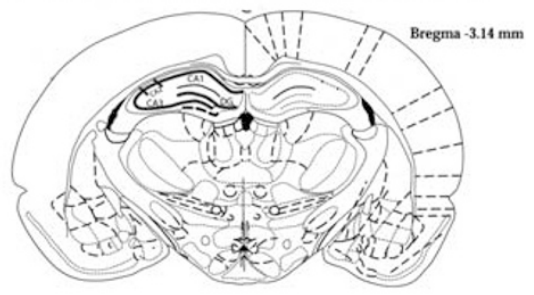

e Dorsal Hippocampus CA1 \& ED1

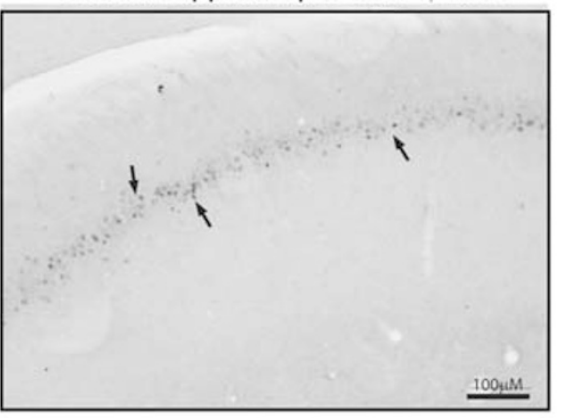

f Dorsal Hippocampus CA3 $\odot$ ED1

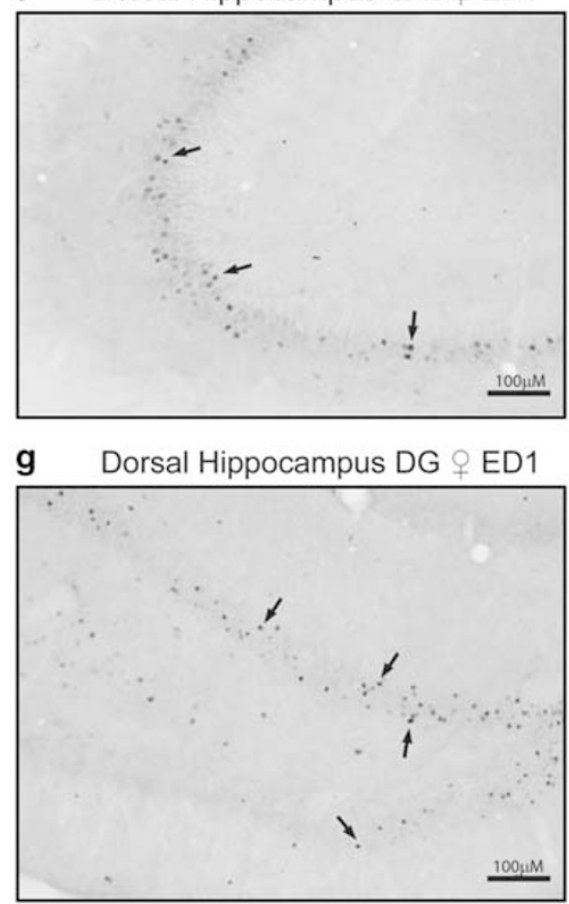

Figure 3 Fos expression in dorsal hippocampus is increased in females on EDI compared with homecage controls. Hippocampus Fos+ neurons were measured in CAI, CA3, and dentate gyrus (DG) in male and female rats following homecage or EDI exposure ( $n=4-8 /$ group). EDI rats were administered saline or S-propranolol ( $10 \mathrm{mg} / \mathrm{kg}$, i.p.) 30 min before EDI testing, and perfused $30 \mathrm{~min}$ following the $90 \mathrm{~min}$ EDI test. Homecage controls were euthanized directly from the homecage at the same time of day as EDI rats. Statistics for all panels are provided in Supplementary Table S2. Two-way ANOVAs were performed between sex and condition followed by post hoc $t$-tests for all analyses. (a) Numbers of Fos + neurons in CAI of male and female homecage, ED I saline, or EDI S-propranolol (EdI S-prop) rats ( $n=4-10 /$ group). There was a significant interaction between sex and test condition on Fos+ neurons in CAI. The post hoc tests revealed that in females, EDI increased Fos+ neuron expression compared with homecage controls, and S-prop reduced Fos+ neuron expression to that of homecage levels; ${ }^{*} p<0.00 \mathrm{I}$. (b) Numbers of Fos+ neurons in CA3 of male and female homecage, EDI saline, or EDI S-prop rats ( $n=4-9 /$ group). There was a significant main effect of test condition on Fos + neurons in CA3. The post hoc tests revealed that EDI +saline male or female rats had increased Fos compared with respective homecage or EDI+S-prop rats; $* p<0.00$ I. (c) Numbers of Fos+ neurons in DG of male and female homecage, EDI saline, or EDI S-prop rats ( $n=4-9 / g r o u p)$. There was a significant main effect of test condition on Fos+ neurons in DG. The post hoc tests revealed male and female rats had significantly higher Fos compared with homecage or S-prop rats; $* 2<0.00 \mathrm{I}$. (d) Depiction of DH areas where Fos was counted, representative image from Bregma - 3.I4 (Paxinos and Watson, 2006). (e) Representative image of Fos expression in DH CAI from an EDI+Saline female. Scale bar $=100 \mu \mathrm{M}$. (f) Representative image of Fos expression in DH CA3 from an ED I +Saline female. Scale bar $=100 \mu M$. (g) Representative image of Fos expression in DH DG from an EDI+Saline female. Scale bar $=100 \mu \mathrm{M}$. 
cocaine-naive subjects (Becker et al, 2016) such that increased Fos during ED1 may reflect a return to baseline Fos levels. Regardless, our findings that Fos levels correlate with ED1 responding in many regions indicate that such activation may play an important role in ED1 drug seeking.

a

Dorsal Raphe (DRD) \%5-HT+/Fos+

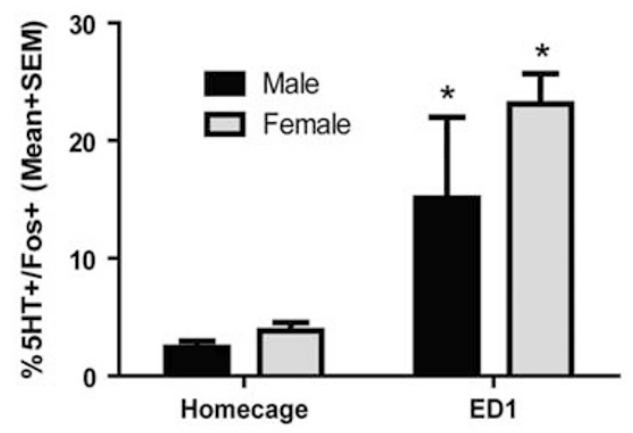

b

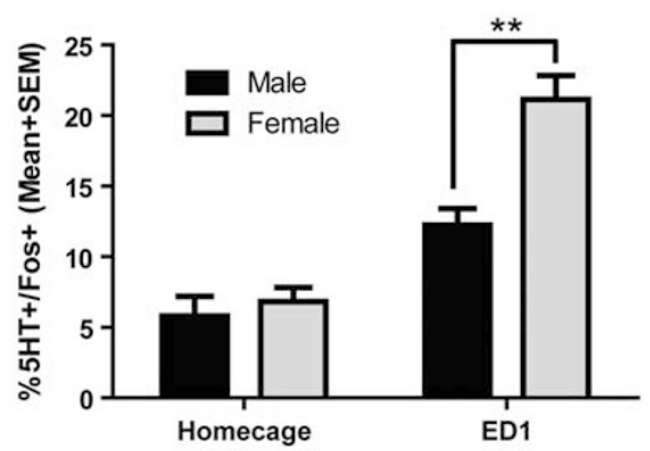

c

Median Raphe (MRN) \%5-HT+/Fos+
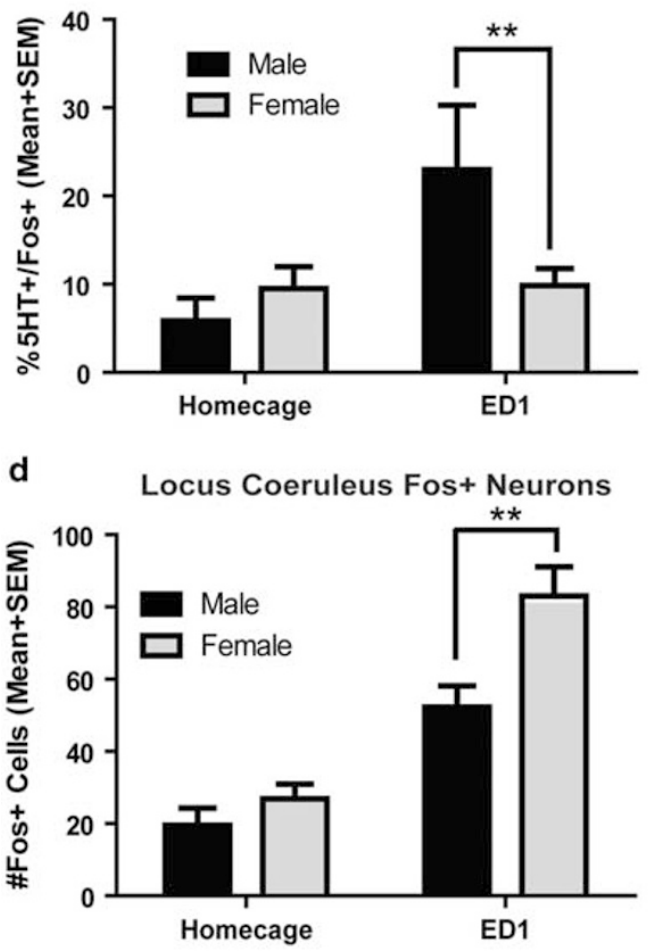

Serotonin cell bodies have distinct projections to the forebrain, depending on their nucleus of origin. DRN, MRN and VLPAG all project to DH, among other targets (Vertes, 2010; Paul et al, 2014; Deakin and Graeff, 1991). Inactivation of DRN by baclofen/muscimol reduced anxiogenic responses

e Depiction of the 5-HT areas where Fos immunoreactive cells were counted

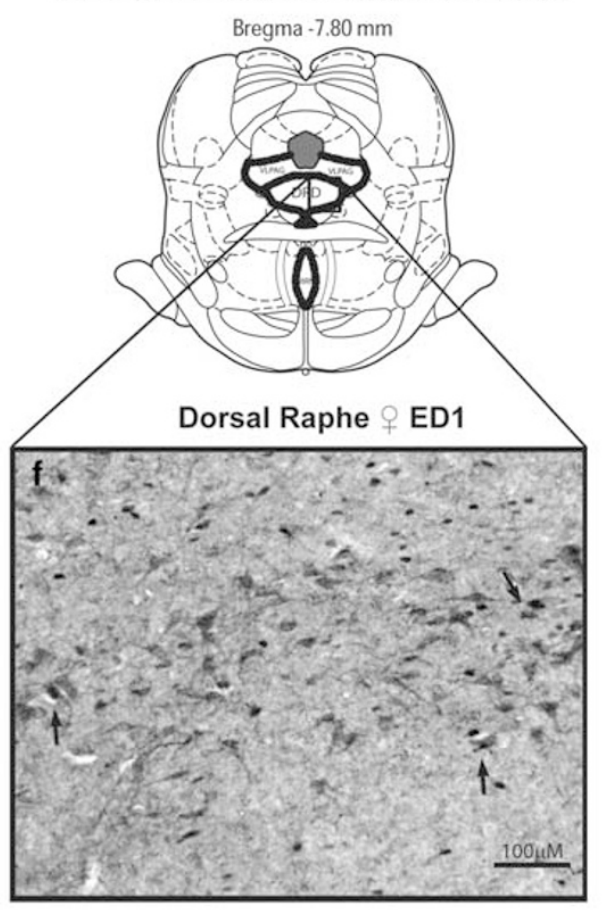

9 Depiction of the LC areas where Fos immunoreactive cells were counted Bregma $-9.72 \mathrm{~mm}$

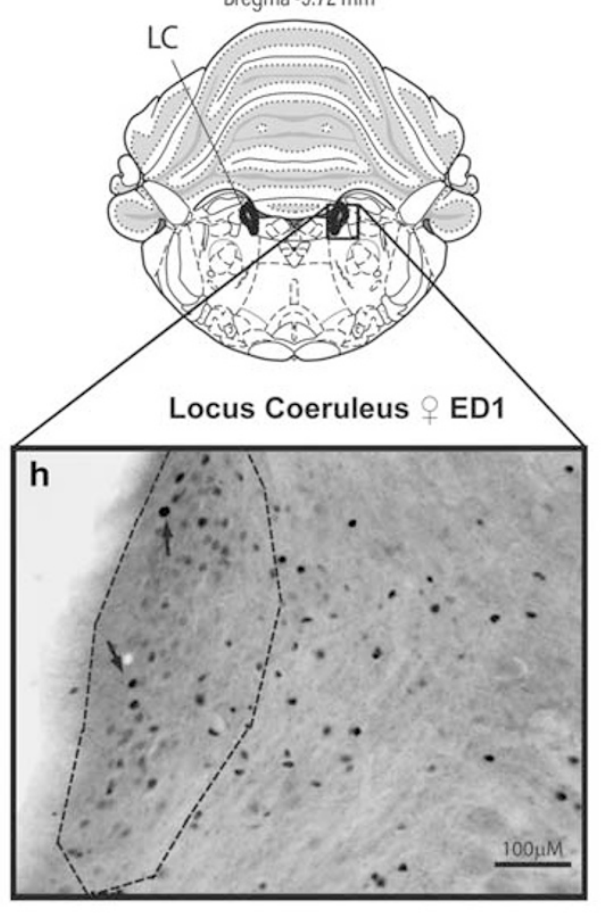

Figure 4 See for caption page on 416. 
of rats during cocaine self-administration, but did not alter motivation for cocaine (Ettenberg et al, 2011). Our studies indicate that DRN is activated on ED1 in both male and female rats, whereas MRN is only activated in males, and VLPAG is primarily activated in females. The percentage of $5-\mathrm{HT}+$ neurons that are Fos+ in DRN, but not MRN or VLPAG, correlated to drug seeking on ED1. These data support the notion that DRN serotonin signaling is involved in initial drug-seeking behaviors.

\section{Propranolol as a Potential Therapeutic: Actions at NE and 5-HT Receptors}

Propranolol reduces cue-induced reinstatement of cocaine seeking and conditioned place preference (CPP) for cocaine in rodents, as well as cue-induced craving in cocaine-dependent humans (Bernardi et al, 2006; Smith and Aston-Jones, 2011; Saladin et al, 2013). Alternatively, systemic propranolol increased dopamine release in NAc, and reduced cocaine selfadministration indicating that propranolol may increase cocaine's reinforcing efficacy (Harris et al, 1996; Perry et al, 2015). Together, these data indicate that propranolol can influence several measures of cocaine abuse. However, these studies were conducted in male subjects, and results were largely attributed to attenuated $\beta$-AR signaling. Our data extend these studies to show involvement of 5-HT1A/1B receptors with propranolol (Pazos et al, 1985) to reduce drug seeking on ED1. We also show that these effects are sex specific, as inhibition of 5-HT1A/1B receptors alone by R-prop was more effective in males than in females, whereas both $\beta$-ARs and 5 -HT1A/1B receptors appear to be important in females.

\section{DH Involvement in ED1 Drug Seeking}

$\mathrm{DH}$ is implicated in context-dependent, but not discrete cueor drug-dependent, cocaine seeking (Fuchs et al, 2005; Luo et al, 2011; Raybuck and Lattal, 2014). As our experimental procedure does not provide discrete cocaine-associated cues or cocaine, we hypothesized that the $\mathrm{DH}$ may be involved in driving drug-seeking behavior on ED1. A recent study showed a role for $\mathrm{DH}$ in both recent (hours-weeks) and remote (weeks-months) drug memories in contrast to nondrug paired memories wherein $\mathrm{DH}$ is required only for recent memories (Raybuck and Lattal, 2014). Our data support this hypothesis, and indicate that $\mathrm{DH}$ is involved in drug seeking on ED1, as DH Fos correlates to ED1 drug seeking, and inhibition of 5 -HT receptors or $\beta$-ARs in $\mathrm{DH}$ reduces ED1 drug seeking. The decreased Fos in $\mathrm{DH}$ following systemic propranolol also correlated with decreased drug seeking. Thus, DH contributes to drug seeking on ED1, possibly via the retrieval of drug-associated contextual memory augmented by the stress of drug absence.

The $\mathrm{DH}$ is implicated in some motivated behaviors (Tracy et al, 2001), but intracranial infusions of a Bet/ICI cocktail or systemic propranolol found no effect on operant responding for sucrose (Leri et al, 2002; Diergaarde et al, 2006), indicating that these manipulations do not cause a general decrease in motivated behaviors. Future studies are needed to address whether our results reflect altered motivation for cocaine.

Previous studies in our lab showed a distinct involvement of CA3 in context-induced reinstatement of cocaine seeking through a CA3-lateral septum-ventral tegmental area (VTA) circuit (Luo et al, 2011). ED1 responding may also involve this circuit, as we observed increased Fos in CA3 on ED1 in both males and females. In contrast to $\mathrm{CA} 3, \mathrm{CA} 1$ receives input from VTA, and sends signals to VTA via the subiculum to drive drug seeking (Lisman and Grace, 2005). Notably, in females only we observed increased Fos in CA1 that correlates to ED1 responding. Other studies have also shown sexually dimorphic Fos expression in $\mathrm{DH}$ following exposure to cocaine-conditioned cues (Zhou et al, 2014). Our results indicate that $\beta$-adrenergic signaling in $\mathrm{DH}$ (perhaps in CA1 in particular) drives increased drug seeking in females compared with males. In the present study, intra-hippocampal cannulae were targeted to CA1, yet 5-HT inhibitors infused into CA1 in males decreased drug-seeking behavior substantially. This could be due to antagonist spread into DG and/or CA3, although extensive ventral spread seems unlikely. Alternatively, 5-HT antagonist infusions into CA1 may interfere with 5-HT modulation of the hippocampus trisynaptic loop, in particular CA3 to CA1 synapses that are involved in associative learning (Gruart et al, 2006). Investigation of these effects is a goal of future studies.

\footnotetext{
Figure 4 Fos expression in serotonin and norepinephrine nuclei is increased on EDI in female and male rats. Fos expression was measured in serotonin and norepinephrine nuclei in male and female rats killed following homecage or EDI exposure; $n=4-7 /$ group. Homecage controls were administered saline and returned to their homecage for $2 \mathrm{~h}$, and killed at the same time of day as EDI rats. EDI rats were administered saline 30 min before EDI testing, and perfused 30 min following the 90 min EDI test. Statistics for all panels are provided in Supplementary Table S2. Two-way ANOVAs were performed between sex and condition followed by post hoc t-tests for all analyses. (a) There was a significant main effect of test condition in the dorsal raphe dorsal area (DRD), wherein post hoc tests revealed the percentage of 5-HT neurons that are Fos+ was significantly increased on EDI in males and females compared with respective homecage controls; * $p<0.05$ ( $n=4-7 /$ group). (b) There was a significant main effect of test condition in the ventrolateral periaqueductal gray (VLPAG), wherein the percentage of 5-HT neurons that are Fos+ is increased on EDI. There was also a significant interaction between test condition and sex, wherein females have a greater increase in EDI Fos compared with males; ${ }^{*} * x<0.05$ ( $\left.n=4-6 / g r o u p\right)$. (c) There was a significant main effect of test condition and sex in the median raphe nucleus (MRN), wherein the percentage of 5-HT neurons that are Fos+ was increased on EDI compared with homecage, and in males compared with females. There was also a significant interaction between test condition and sex, wherein females had a greater increase in EDI Fos compared with males; *** $p<0.05$ ( $n=4-7 / g r o u p)$. (d) There was a significant main effect of test condition and sex in the locus coeruleus, wherein the number of Fos+ neurons was increased on EDI compared with homecage subjects, and increased in females compared with males. There was also a significant interaction between test condition and sex, wherein females have a greater increase in EDI Fos compared with males; *** $<0.05$ ( $n=4-$ I I/group). (e) Depiction of serotonin cell body regions where Fos was counted, representative section from Bregma - 7.80 (Paxinos and Watson, 2006). (f) Representative image of Fos and 5-HT expression in DRD from an EDI +saline female; scale bar $=100 \mu \mathrm{M}$. (g) Depiction of LC region where Fos was counted, representative section from Bregma - 9.72 (Paxinos and Watson, 2006). (h) Representative image of Fos expression in LC from an EDI +Saline female. Scale bar $=100 \mu M$.
} 


\section{NE and 5-HT Signaling May Converge on Stress Pathways to Influence Drug-Seeking Behavior}

Our results may involve brain stress circuits, consistent with our hypothesis that ED1 represents a stressful event due to the abstinence of expected cocaine. Among cocainedependent patients, exposure to drug-associated cues or stressors results in increased adrenocorticotrophic hormone $(\mathrm{ACTH})$, cortisol, and hypothalamic-pituitary-adrenal (HPA) axis activity (Sinha et al, 2000, 2003). Among abstinent heroin addicts and cocaine-dependent individuals, propranolol is effective in reducing cortisol-, stress-, or cueinduced cravings (Childress et al, 1999; Zhao et al, 2010; Saladin et al, 2013). Racemic propranolol antagonizes $\beta$-ARs and 5-HT receptors, and therefore these effects may be due to the actions of either of these receptors. In rodents, conflicting reports indicate that anxiety behavior after cocaine withdrawal may occur in a relatively narrow timeframe, or up to a period of at least 6 weeks following abstinence (Harris et al, 2001; Chartoff and Carlezon, 2014). Anxiety produced by cocaine withdrawal may contribute to drug seeking via a stress-dependent mechanism. Consistent with this possibility, we found that ED1 exposure increased corticosterone levels compared with baseline or homecage levels in males and females, and correlated to ED1 drugseeking behavior. In addition, there is sex-specific involvement of $\beta$-ARs and 5-HT receptors in driving drug seeking on ED1 that may involve stress.

\section{SUMMARY}

Our studies indicate sexually divergent roles for hippocampal $\beta$-ARs and 5-HT receptors in cocaine-seeking behavior in early abstinence that may involve the stress of drug absence. We hypothesize that reducing such ED1-associated stress and drug seeking may promote more successful maintenance of abstinence by decreasing stress-induced negative reinforcement and aversive memories of abstinence. Future studies aim to identify the effects of reducing stress and manipulating ED1 drug seeking on later propensity for drug relapse. Thus, these data represent novel findings of the neurobiological basis of sex-specific phenotypes associated with cocaine seeking that may inform treatment programs.

\section{FUNDING AND DISCLOSURE}

This research received project support from PHS grant P50 DA016511. The authors declare no conflict of interest.

\section{ACKNOWLEDGMENTS}

We thank Jennifer Osborne for her assistance with behavioral testing and Mark Presker for his assistance with tail vein blood draws.

\section{REFERENCES}

Babstock D, Malsbury CW, Harley CW (1997). The dorsal locus coeruleus is larger in male than in female Sprague-Dawley rats. Neurosci Lett 224: 157-160.

Becker JA, Kieffer BL, Le Merrer J (2016). Differential behavioral and molecular alterations upon protracted abstinence from cocaine versus morphine, nicotine, THC and alcohol. Addict Biol (e-pub ahead of print).

Becker JB, Hu M (2008). Sex differences in drug abuse. Front Neuroendocrinol 29: 36-47.

Bernardi RE, Lattal KM, Berger SP (2006). Postretrieval propranolol disrupts a cocaine conditioned place preference. Neuroreport 17: 1443-1447.

Buffalari DM, Baldwin CK, Feltenstein MW, See RE (2012). Corticotrophin releasing factor (CRF) induced reinstatement of cocaine seeking in male and female rats. Physiol Behav 105: 209-214.

Chartoff EH, Carlezon WA Jr (2014). Drug withdrawal conceptualized as a stressor. Behav Pharmacol 25: 473-492.

Childress AR, Mozley PD, McElgin W, Fitzgerald J, Reivich M, O'Brien CP (1999). Limbic activation during cue-induced cocaine craving. Am J Psychiatry 156: 11-18.

Curtis AL, Bethea T, Valentino RJ (2006). Sexually dimorphic responses of the brain norepinephrine system to stress and corticotropinreleasing factor. Neuropsychopharmacology 31: 544-554.

Dahlstrom A, Fuxe K (1964). Localization of monoamines in the lower brain stem. Experientia 20: 398-399.

Deakin J, Graeff F (1991). 5-HT and mechanisms of defence. J Psychopharmacol 5: 305-315.

Diergaarde L, Schoffelmeer AN, De Vries TJ (2006). Betaadrenoceptor mediated inhibition of long-term reward-related memory reconsolidation. Behav Brain Res 170: 333-336.

Ettenberg A, Ofer OA, Mueller CL, Waldroup S, Cohen A, Ben-Shahar O (2011). Inactivation of the dorsal raphe nucleus reduces the anxiogenic response of rats running an alley for intravenous cocaine. Pharmacol Biochem Behav 97: 632-639.

Feltenstein MW, Henderson AR, See RE (2011). Enhancement of cue-induced reinstatement of cocaine-seeking in rats by yohimbine: sex differences and the role of the estrous cycle. Psychopharmacology 216: 53-62.

Feltenstein MW, See RE (2007). Plasma progesterone levels and cocaine-seeking in freely cycling female rats across the estrous cycle. Drug Alcohol Depend 89: 183-189.

Fuchs RA, Evans KA, Ledford CC, Parker MP, Case JM, Mehta RH et al (2005). The role of the dorsomedial prefrontal cortex, basolateral amygdala, and dorsal hippocampus in contextual reinstatement of cocaine seeking in rats. Neuropsychopharmacology 30: 296-309.

Galea L, McEwen B, Tanapat P, Deak T, Spencer R, Dhabhar F (1997). Sex differences in dendritic atrophy of CA3 pyramidal neurons in response to chronic restraint stress. Neuroscience $\mathbf{8 1}$ : 689-697.

Griffin ML, Weiss RD, Mirin SM, Lange U (1989). A comparison of male and female cocaine abusers. Arch Gen Psychiatry 46: 122-126.

Gruart A, Munoz MD, Delgado-Garcia JM (2006). Involvement of the CA3-CA1 synapse in the acquisition of associative learning in behaving mice. J Neurosci 26: 1077-1087.

Harris GC, Altomare K, Aston-Jones G (2001). Preference for a cocaine-associated environment is attenuated by augmented accumbal serotonin in cocaine withdrawn rats. Psychopharmacology 156: 14-22.

Harris GC, Aston-Jones G (1993). Beta-adrenergic antagonists attenuate withdrawal anxiety in cocaine- and morphinedependent rats. Psychopharmacology 113: 131-136.

Harris GC, Aston-Jones G (2001). Augmented accumbal serotonin levels decrease the preference for a morphine associated environment during withdrawal. Neuropsychopharmacology 24: 75-85.

Harris GC, Hedaya MA, Pan WJ, Kalivas P (1996). beta-adrenergic antagonism alters the behavioral and neurochemical responses to cocaine. Neuropsychopharmacology 14: 195-204.

Howerton AR, Roland AV, Fluharty JM, Marshall A, Chen A, Daniels D et al (2014). Sex differences in corticotropin-releasing 
factor receptor-1 action within the dorsal raphe nucleus in stress responsivity. Biol Psychiatry 75: 873-883.

Kampman KM (2009). New medications for the treatment of cocaine dependence. Ann Ist Super Sanita 45: 109-115.

Kippin TE, Fuchs RA, Mehta RH, Case JM, Parker MP, BimonteNelson HA et al (2005). Potentiation of cocaine-primed reinstatement of drug seeking in female rats during estrus. Psychopharmacology 182: 245-252.

Kosten TA, Gawin FH, Kosten TR, Rounsaville BJ (1993). Gender differences in cocaine use and treatment response. J Subst Abuse Treat 10: 63-66.

Le Moal M, Koob GF (2007). Drug addiction: pathways to the disease and pathophysiological perspectives. Eur Neuropsychopharmacol 17: 377-393.

Leri F, Flores J, Rodaros D, Stewart J (2002). Blockade of stress-induced but not cocaine-induced reinstatement by infusion of noradrenergic antagonists into the bed nucleus of the stria terminalis or the central nucleus of the amygdala. J Neurosci 22: 5713-5718.

Lisman JE, Grace AA (2005). The hippocampal-VTA loop: controlling the entry of information into long-term memory. Neuron 46: 703-713.

Lu J, Wu XY, Zhu QB, Li J, Shi LG, Wu JL et al (2015). Sex differences in the stress response in SD rats. Behav Brain Res 284: 231-237.

Luo AH, Tahsili-Fahadan P, Wise RA, Lupica CR, Aston-Jones G (2011). Linking context with reward: a functional circuit from hippocampal CA3 to ventral tegmental area. Science 333: 353-357.

Lynch WJ, Taylor JR (2004). Sex differences in the behavioral effects of 24-h/day access to cocaine under a discrete trial procedure. Neuropsychopharmacology 29: 943-951.

Madeira MD, Paula-Barbosa MM (1993). Reorganization of mossy fiber synapses in male and female hypothyroid rats: a stereological study. J Comp Neurol 337: 334-352.

Mahler SV, Aston-Jones GS (2012). Fos activation of selective afferents to ventral tegmental area during cue-induced reinstatement of cocaine seeking in rats. J Neurosci 32: 13309-13326.

O'Brien CP, Childress AR, McLellan AT, Ehrman R (1992). Classical conditioning in drug-dependent humans. Ann NY Acad Sci 654: 400-415.

Paul ED, Hale MW, Lukkes JL, Valentine MJ, Sarchet DM, Lowry CA (2011). Repeated social defeat increases reactive emotional coping behavior and alters functional responses in serotonergic neurons in the rat dorsal raphe nucleus. Physiol Behav 104: 272-282.

Paxinos G, Watson C (2006). The Rat Brain in Stereotaxic Coordinates 6th Edition. Elsevier.

Pazos A, Engel G, Palacios JM (1985). beta-Adrenoceptor blocking agents recognize a subpopulation of serotonin receptors in brain. Brain Res 343: 403-408.

Perry AN, Westenbroek C, Jagannathan L, Becker JB (2015). The roles of dopamine and alpha1-adrenergic receptors in cocaine preferences in female and male rats. Neuropsychopharmacology 40: $2696-2704$.

Raybuck JD, Lattal KM (2014). Differential effects of dorsal hippocampal inactivation on expression of recent and remote drug and fear memory. Neurosci Lett 569: 1-5.

Rhodes ME, Rubin RT (1999). Functional sex differences ('sexual diergism') of central nervous system cholinergic systems, vasopressin, and hypothalamic-pituitary-adrenal axis activity in mammals: a selective review. Brain Res Rev 30: 135-152.

Robbins SJ, Ehrman RN, Childress AR, O'Brien CP (1999). Comparing levels of cocaine cue reactivity in male and female outpatients. Drug Alcohol Depend 53: 223-230.
Saladin ME, Gray KM, McRae-Clark AL, Larowe SD, Yeatts SD, Baker NL et al (2013). A double blind, placebo-controlled study of the effects of post-retrieval propranolol on reconsolidation of memory for craving and cue reactivity in cocaine dependent humans. Psychopharmacology 226: 721-737.

Shors T, Chua C, Falduto J (2001). Sex differences and opposite effects of stress on dendritic spine density in the male versus female hippocampus. J Neurosci 21: 6292-6297.

Sinha R (2001). How does stress increase risk of drug abuse and relapse? Psychopharmacology 158: 343-359.

Sinha R (2008). Chronic stress, drug use, and vulnerability to addiction. Ann NY Acad Sci 1141: 105-130.

Sinha R, Fuse T, Aubin LR, O'Malley SS (2000). Psychological stress, drug-related cues and cocaine craving. Psychopharmacology 152: 140-148.

Sinha R, Talih M, Malison R, Cooney N, Anderson GM, Kreek MJ (2003). Hypothalamic-pituitary-adrenal axis and sympathoadreno-medullary responses during stress-induced and drug cue-induced cocaine craving states. Psychopharmacology 170: $62-72$.

Smith RJ, Aston-Jones G (2008). Noradrenergic transmission in the extended amygdala: role in increased drug-seeking and relapse during protracted drug abstinence. Brain Struct Funct 213: 43-61.

Smith RJ, Aston-Jones G (2011). alpha(2) Adrenergic and imidazoline receptor agonists prevent cue-induced cocaine seeking. Biol Psychiatry 70: 712-719.

Smith RJ, See RE, Aston-Jones G (2009). Orexin/hypocretin signaling at the orexin 1 receptor regulates cue-elicited cocaine-seeking. Eur J Neurosci 30: 493-503.

Sofuoglu M, Brown S, Babb DA, Pentel PR, Hatsukami DK (2000). Carvedilol affects the physiological and behavioral response to smoked cocaine in humans. Drug Alcohol Depend 60: 69-76.

Tinnikov A (1999). Responses of serum corticosterone and corticosteroid-bining globulin to acute and prolonged stress in the rat. Endocrine 11: 145-150.

Tracy AL, Jarrard LE, Davidson TL (2001). The hippocampus and motivation revisited: appetite and activity. Behav Brain Res 127: $13-23$.

Turner BB (1992). Sex differences in the binding of type I and type II corticosteroid receptors in rat hippocampus. Brain Res 581: 229-236.

Vertes RP. Serotonergic regulation of rhythmical activity of the brain, concentrating on the hippocampus. Handbook of the Behavioral Neurobiology of Serotonin. In: Muller CP, Jacobs BL (eds). Academic Press: New York, 2010, pp 277-292.

Weiss R, Griffin M, Hufford C, Muenz L, Najavits L, Jansson S et al (2010). Early prediction of initiation of abstinence from cocaine. Am J Addict 6: 224-231.

Wikler A (1973). Dynamics of drug dependence. Implications of a conditioning theory for research and treatment. Arch Gen Psychiatry 28: 611-616.

Wirtshafter D, Asin KE, Lorens SA (1986). Serotoninimmunoreactive projections to the hippocampus from the interpeduncular nucleus in the rat. Neurosci Lett 64: 259-262.

Zhao LY, Shi J, Zhang XL, Epstein DH, Zhang XY, Liu Y et al (2010). Stress enhances retrieval of drug-related memories in abstinent heroin addicts. Neuropsychopharmacology 35: $720-726$.

Zhou L, Ghee SM, Chan C, Lin L, Cameron MD, Kenny PJ et al (2012). Orexin-1 receptor mediation of cocaine seeking in male and female rats. J Pharmacol Exp Ther 340: 801-809.

Zhou L, Pruitt C, Shin CB, Garcia AD, Zavala AR, See RE (2014). Fos expression induced by cocaine-conditioned cues in male and female rats. Brain StructFunct 219: 1831-1840.

Supplementary Information accompanies the paper on the Neuropsychopharmacology website (http://www.nature.com/npp) 